\title{
Lithium abundances in metal-poor stars
}

\section{New observations ${ }^{\star}$}

\author{
C.M. Gutiérrez ${ }^{1}$, R.J. García López ${ }^{1}$, R. Rebolo ${ }^{1,2}$, E.L. Martín ${ }^{1,3}$, and P. François ${ }^{4}$ \\ 1 Instituto de Astrofísica de Canarias, E-38200 La Laguna, Tenerife, Spain \\ 2 Consejo Superior de Investigaciones Científicas, Spain \\ 3 Astronomy Department, University of California at Berkeley, 601 Campbell Hall, Berkeley, CA 94720, U.S.A. \\ ${ }^{4}$ Observatoire de Paris, DASGAL, URA 335 du CNRS, 61 Av. de l'Observatoire, F-75014 Paris, France
}

Received January 23, 1998; accepted March 1, 1999

\begin{abstract}
We present the lithium measurements of a continuing programme of light element abundances in metalpoor stars. New equivalent widths of the Li I $\lambda 670.8 \mathrm{~nm}$ resonance line in 67 metal-poor stars covering the metallicity range $-3.5 \leq[\mathrm{Fe} / \mathrm{H}] \leq-0.4$ are reported. For about half of this sample, the observations presented here represent the first measurement of the Li I line. The sample allowed a statistical comparison with previous measurements from other authors and a study of the consistency and reliability of the quoted error bars. This paper shows that for most of the stars these error bars are good estimates of the true uncertainties associated with the determination of the equivalent widths of the $\mathrm{Li}$ I line. However, about $20 \%$ of the stars with two or more independent measurements show discrepancies in the Li I equivalent widths; in these cases, other sources of uncertainty not properly taken into account (binarity effects, cosmic rays, imperfect flat-field correction, continuum determination, etc.) could also be important. Conclusions on the possible lithium abundance trends versus effective temperature or metallicity and on any intrinsic scatter should be treated cautiously until their robustness vis-à-vis these additional uncertainties is proved.
\end{abstract}

Key words: Galaxy: evolution — stars: abundances — stars: late-type — stars: Population II

Send offprint requests to: R. Rebolo (rrl@iac.es)

* Based on observations made with the Isaac Newton and Nordic Optical Telescopes, which are operated on the island of La Palma by the Isaac Newton Group and the NOT Scientific Association, respectively, in the Spanish Observatorio del Roque de los Muchachos of the Instituto de Astrofísica de Canarias.

\section{Introduction}

According to the standard big bang nucleosynthesis model, lithium is one of the few elements synthesized in the first minutes of the Universe. In this scenario the primordial synthesis of lithium is very sensitive to the baryon/photon ratio $\left(n_{\mathrm{b}} / n_{\gamma}\right)$, and the astronomical determination of its primordial abundance can constrain the baryonic contribution to the density of the Universe. Since the discovery of a rather uniform lithium abundance, the so called lithium plateau, in the hotter halo dwarfs (Spite \& Spite 1982; Rebolo et al. 1988) at about a value $\log n(\mathrm{Li})=2$ (on the usual scale where $\log n(\mathrm{H})=12$ ), there has been a long debate on whether or not this abundance reflects the primordial one. Some theoretical models suggest that processes such as diffusion, rotational mixing etc. may have depleted the initial lithium abundance of the hotter $\left(T_{\text {eff }} \geq\right.$ $5600 \mathrm{~K}$ ) halo dwarfs and reduced it to its present atmospheric value. The fingerprint of such a process has been searched and claimed to be possibly manifest in a trend of the $\mathrm{Li}$ abundance versus $[\mathrm{Fe} / \mathrm{H}]$ and $T_{\text {eff }}$ (Thorburn 1994; Norris et al. 1994; Ryan et al. 1996), or in a spread of the lithium abundance around the plateau (Deliyannis et al. 1993, 1995).

The observational evidence for a global depletion process acting on these halo dwarfs is not conclusive. Molaro et al. (1995) using a reddening-free temperature scale and Bonifacio \& Molaro (1997) using direct infrared flux method temperatures provided by Alonso et al. (1996) have made a bivariate analysis of a large sample of halo dwarfs, and conclude in both cases that there is no evidence of these trends versus metallicity and effective temperature. The same conclusion has been reached by Spite et al. (1996), who re-analysed an important part of the current observations using temperatures determined from several independent methods; these authors propose that 

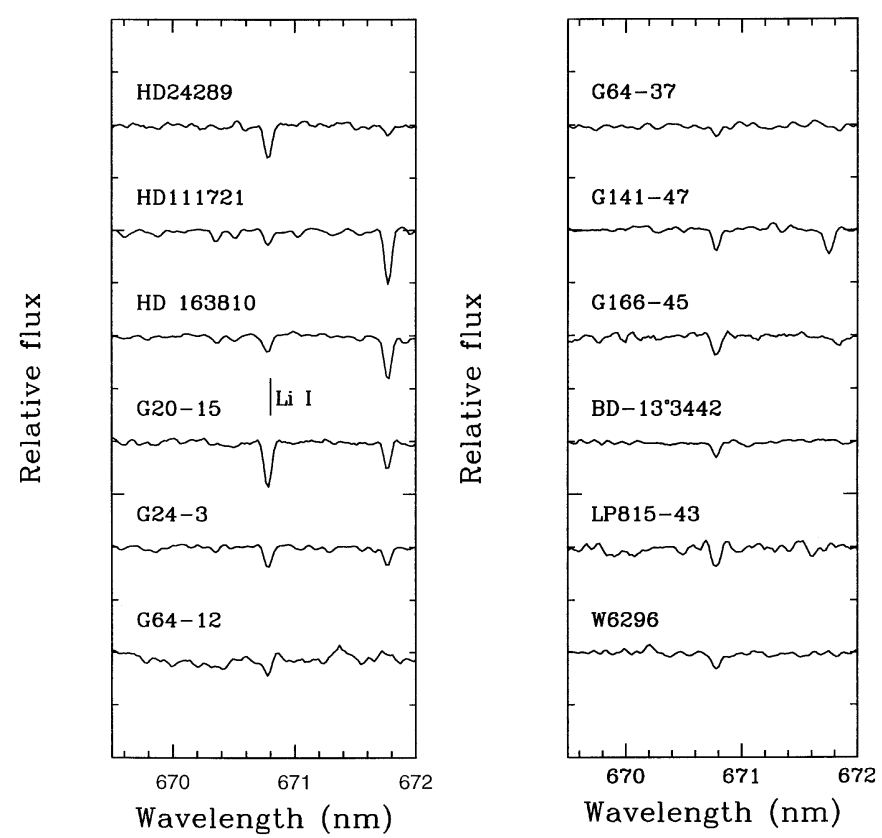

Fig. 1. Spectra of several stars of our sample in the Li I $\lambda$ $670.8 \mathrm{~nm}$ region. A three pixels box-car smoothing was applied to each spectrum

the observed spread around the plateau is due to error measurements and uncertainties in effective temperatures. On the other hand, Ryan et al. (1996) used a temperature scale similar to the scale of Carney et al. (1994) and in this case, a multiple-regression analysis shows that the correlations actually exist, especially when the most metal-poor stars are included in the sample.

This is the first paper of a series that intend to revisit the lithium problem in halo dwarfs. The paper presents new Li I $\lambda 670.8 \mathrm{~nm}$ observations in 67 metal-poor stars. Here, the observations and the comparison with other works are presented. The main analyses and implications will be given in a forthcoming paper.

\section{Observations and data reduction}

The observations presented here were obtained as part of an international collaboration at the observatories of the Canary Islands in several campaigns during the years 1990 and 1991. We used the Cassegrain foci of the $2.5 \mathrm{~m}$ Isaac Newton (INT) Telescope and Nordic Optical (NOT) Telescope at the Observatorio del Roque de los Muchachos (La Palma). Spectra in the region of the Li I line were obtained using the Intermediate Dispersion Spectrograph (IDS) and the IACUB echelle spectrograph at the INT and NOT, respectively. The $\mathrm{H} 1800 \mathrm{~V}$ grating and the $500 \mathrm{~mm}$ camera with a $385 \times 578$ GEC-CCD were used at the IDS, providing a dispersion of $0.22 \AA$ per pixel. The adopted slit-width was usually 1 arcsec giving an effective resolution of $0.44 \AA$. The IACUB echelle spectrograph (McKeith et al. 1993) was used with a Thomson $1024 \times 1024$ CCD
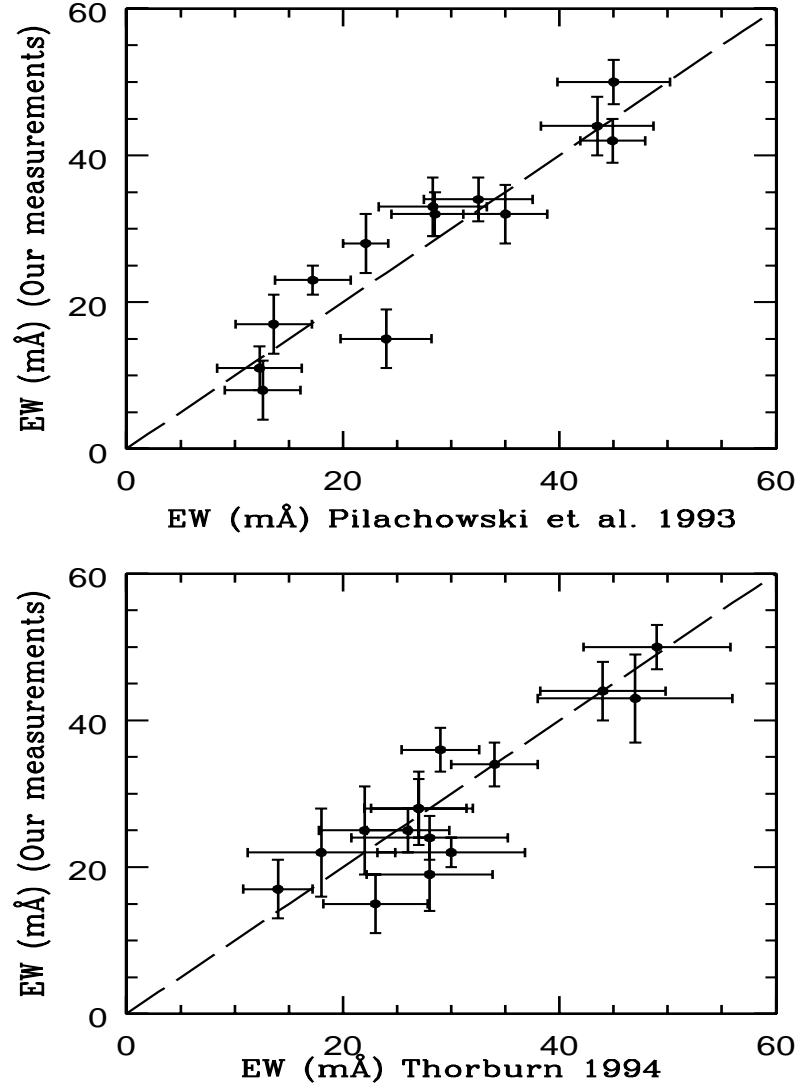

Fig. 2. Comparison between our measurements of the LiI $\lambda 670.8 \mathrm{~nm}$ equivalent widths and those by Pilachowski et al. (1993; top) and Thorburn (1994; down). The error bars represent the estimated $95 \%$ confidence limits

binned in the spectral direction to provide a dispersion of $0.1 \AA$ per pixel. The 0.7 arcsec slit width employed gave a final effective resolution of $\sim 0.22 \AA$ (in the case of G18147 we used a 1 arcsec slit which gave $0.30 \AA$ ). Typically, we recorded spectra with exposure times $\sim 1800$ s. For the fainter stars, two or more exposures were combined in order to have a homogeneous sample with signal to noise in the range $100-200$. Using the same instrumental configuration, several flat-field lamps were recorded each night. For wavelength calibration $\mathrm{Cu}-\mathrm{Ar}$ lamp spectra were used.

Most of the objects were selected from the sample of metal-poor stars in the works of Carney et al. (1987), Laird et al. (1988), and Schuster \& Nissen (1988, 1989). Table 1 lists the stars observed, telescopes used, epoch of observation, exposure times and visual magnitudes. Several typical spectra in the region of the LiI line are plotted in Fig. 1. Table 2 lists the relevant photometric data and metallicities which will be used in a forthcoming paper to determine the stellar parameters $T_{\text {eff }}$ and $\log g$ needed for the abundance analysis. Photometric magnitudes were obtained from the literature and the SIMBAD data base. Metallicities were also obtained from the literature using mainly the compilations by Schuster \& Nissen 
Table 1. Programme stars

\begin{tabular}{|c|c|c|c|c|c|}
\hline Star & Other designations & Telescope & Epoch & $t_{\exp }(\mathrm{s})$ & $V$ \\
\hline HD 4906 & $\mathrm{G} 32-53, \mathrm{BD}+18^{\circ} 111$ & NOT & 1991 Aug. & 1200 & 8.76 \\
\hline HD 24289 & $\mathrm{G} 80-28, \mathrm{BD}-04^{\circ} 680$ & INT & 1991 Jan. & $2 \times 1500$ & 9.977 \\
\hline HD 45282 & $\mathrm{BD}+3^{\circ} 1247$ & INT & 1991 Jan. & 1000 & 8.028 \\
\hline HD 88725 & G44-6 & INT & 1991 May & 1200 & 7.750 \\
\hline HD 101063 & & INT & 1991 Мау & 1400 & 9.440 \\
\hline HD 103912 & $\mathrm{G} 122-57, \mathrm{BD}+49^{\circ} 2098$ & INT & 1991 May & 800 & 8.360 \\
\hline HD 106038 & $\mathrm{G} 12-21, \mathrm{BD}+14^{\circ} 2481$ & INT & 1991 Jan. & $2 \times 1500$ & 10.179 \\
\hline HD 108976 & $\mathrm{BD}+28^{\circ} 2125$ & INT & 1991 Jan. & 1200 & 8.61 \\
\hline HD 111721 & $\mathrm{BD}-12^{\circ} 3709$ & INT & 1991 May & 600 & 7.988 \\
\hline HD 112573 & G61-24 & INT & 1991 May & $1200+1145$ & 8.97 \\
\hline HD 126681 & $\mathrm{BD}-17^{\circ} 4092$ & INT & 1991 May & 1200 & 9.300 \\
\hline HD 134439 & $\mathrm{BD}-15^{\circ} 4042$ & INT & 1991 May & 1200 & 9.058 \\
\hline HD 134440 & $\mathrm{BD}-15^{\circ} 4041$ & INT & 1991 May & 1200 & 9.419 \\
\hline HD 149414 & $\mathrm{G} 17-25, \mathrm{BD}+53^{\circ} 1871$ & INT & 1991 May & $1200+1000$ & 9.611 \\
\hline HD 157948 & $\mathrm{G} 182-7, \mathrm{BD}+38^{\circ} 2932$ & INT & 1991 May & $2 \times 1200$ & 8.095 \\
\hline HD 158226 & $\mathrm{G} 181-47, \mathrm{BD}+31^{\circ} 3027$ & NOT & 1991 Aug. & $2 \times 1200$ & 8.500 \\
\hline HD 161770 & $\mathrm{G} 154-21, \mathrm{BD}-9^{\circ} 4604$ & INT & 1991 May & $2 \times 1300$ & 9.66 \\
\hline HD 163810 & $\mathrm{G} 154-36, \mathrm{BD}-13^{\circ} 4807$ & INT & 1991 May & $1300+1000$ & 9.635 \\
\hline HD 179626 & $\mathrm{G} 22-20, \mathrm{BD}-00^{\circ} 3676$ & INT & 1991 May & 1200 & 9.210 \\
\hline HD 184448 & G229-34 & NOT & 1991 Aug. & $600+1000$ & 8.050 \\
\hline HD 199476 & G261-29 & NOT & 1991 Aug. & $2 \times 600$ & 7.810 \\
\hline HD 200580 & G25-15 & INT & 1991 May & 250 & 7.340 \\
\hline HD 204155 & G25-29 & NOT & 1991 Aug. & 1200 & 8.500 \\
\hline HD 215065 & $\mathrm{G} 241-18, \mathrm{BD}+65^{\circ} 1796$ & NOT & 1991 Aug. & $2 \times 1200$ & 7.460 \\
\hline HD 215257 & $\mathrm{G} 27-44, \mathrm{BD}+03^{\circ} 4763$ & NOT & 1991 Aug. & 1800 & 7.420 \\
\hline HD 216588 & G157-21 & NOT & 1991 Aug. & 1200 & 9.270 \\
\hline HD 218209 & $\mathrm{G} 241-42, \mathrm{BD}+67^{\circ} 1498$ & NOT & 1991 Aug. & $2 \times 1200$ & 7.480 \\
\hline HD 221613 & $\mathrm{G} 171-3, \mathrm{BD}+42^{\circ} 4700$ & NOT & 1991 Aug. & $2 \times 600$ & 7.140 \\
\hline G3-16 & $\mathrm{BD}+04^{\circ} 302$ & INT & 1990 Oct. & $1900+1500$ & 10.507 \\
\hline G16-9 & $\mathrm{BD}+05^{\circ} 3080$ & INT & 1991 May & $1200+800$ & 9.140 \\
\hline G20-15 & $\mathrm{BD}-08^{\circ} 4501$ & INT & 1991 May & 1500 & 10.591 \\
\hline \multirow[t]{2}{*}{ G24-3 } & & INT & 1990 Oct. & $1800+1200$ & 10.467 \\
\hline & & & 1991 May & $1500+1400$ & \\
\hline G26-9 & $\mathrm{BD}-00^{\circ} 4234$ & INT & 1990 Oct. & 120 & 9.798 \\
\hline G29-23 & $\mathrm{BD}+02^{\circ} 4651$ & INT & 1990 Oct. & $500+1700$ & 10.230 \\
\hline G41-41 & $\mathrm{BD}+9^{\circ} 2190$ & INT & 1991 May & $2 \times 1500+400$ & 11.14 \\
\hline
\end{tabular}

(1989), and Carney et al. (1987). In general, the differences between these metallicities for the objects in common are of order $0.1-0.2$ dex, but in a few of them (the most metal-poor stars) these differences can be as large as 1 dex.

All the images were processed with the $\operatorname{IRAF}^{1}$ package following standard techniques of bias subtraction, flat-field corrections, optimal spectrum extraction and sky subtraction, wavelength calibration and linearization, and continuum normalization. The identification of the Li I $\lambda 670.8 \mathrm{~nm}$ line was performed on the basis of the radial velocities measured by Laird et al. (1988), and Carney \& Latham (1987), and/or the relative positions of the Fe I $\lambda 667.8$ and Ca I $\lambda 671.7 \mathrm{~nm}$ lines. An unambiguous

\footnotetext{
${ }^{1}$ IRAF is distributed by National Optical Astronomical Observatories, which is operated by the Association of Universities for Research in Astronomy, Inc., under contract with the National Science Foundation, U.S.A.
}

identification of the Li I spectral feature was always possible for the stars in Table 1. Those few very metal-poor stars in our original sample with poor signal to noise ratio and nearly featureless spectra for which the radial velocity was unknown have not been included in this paper.

\section{Equivalent widths}

The equivalent widths (EWs) were measured by direct integration under the continuum using the subroutine SPLOT of the IRAF package. They were determined independently by three of us to minimize the effects of a subjective location of the continuum. The finally adopted EWs and the quoted errorbars result from a critical discussion of these determinations. Table 2 presents the resulting Li I EWs with the estimated 95\% confidence limit error bars. The uncertainties are in general $\leq 5 \mathrm{m \AA}$. 
Table 1. continued

\begin{tabular}{|c|c|c|c|c|c|}
\hline Star & Other designations & Telescope & Epoch & $t_{\exp }(\mathrm{s})$ & $V$ \\
\hline G59-27 & $\mathrm{BD}+28^{\circ} 2137$ & INT & 1991 May & $3 \times 1500$ & 10.892 \\
\hline G60-26 & $\mathrm{BD}+13^{\circ} 2567$ & INT & 1991 May & 1000 & 9.813 \\
\hline G63-46 & $\mathrm{BD}+13^{\circ} 2698$ & INT & 1991 Jan. & 1500 & 9.378 \\
\hline G64-12 & & INT & 1991 May & $4 \times 1500$ & 11.458 \\
\hline G64-37 & & INT & 1991 May & $\begin{array}{l}5 \times 1500 \\
2 \times 1600\end{array}$ & 11.144 \\
\hline G66-22 & $\mathrm{BD}+06^{\circ} 2932$ & INT & 1991 May & $2 \times 1600+1200$ & 10.47 \\
\hline G69-4A & HD3266 & NOT & 1991 Aug. & $2 \times 1200$ & 7.980 \\
\hline G69-4B & & NOT & 1991 Aug. & $2 \times 1200$ & 7.980 \\
\hline G74-5 & $\mathrm{BD}+29^{\circ} 366$ & NOT & 1991 Aug. & $2 \times 1200$ & 8.771 \\
\hline G88-27 & $\mathrm{BD}+19^{\circ} 1730$ & INT & 1991 May & $1200+1500$ & 10.712 \\
\hline G88-32 & $\mathrm{BD}+24^{\circ} 1676$ & INT & 1990 Oct. & $2100+1500$ & 10.780 \\
\hline G90-3 & & INT & 1990 Oct. & 1500 & 10.397 \\
\hline G101-34 & $\mathrm{BD}+38^{\circ} 1456$ & INT & 1990 Oct. & $2 \times 1500$ & 10.730 \\
\hline G123-9 & $\mathrm{BD}+44^{\circ} 2166$ & INT & 1991 May & $2 \times 1400$ & 10.500 \\
\hline G125-13 & $\mathrm{BD}+35^{\circ} 3659 \mathrm{~s}$ & INT & 1991 May & 1200 & 10.238 \\
\hline G125-64 & $\mathrm{BD}+42^{\circ} 3607$ & INT & 1991 May & 1500 & 10.110 \\
\hline G140-46 & $\mathrm{BD}+05^{\circ} 3640$ & INT & 1991 May & $1500+1600$ & 10.348 \\
\hline G141-19 & $\mathrm{BD}+13^{\circ} 3683$ & INT & 1991 May & $\begin{array}{l}2 \times 1500 \\
2 \times 1200+1400\end{array}$ & 10.582 \\
\hline G141-47 & $\mathrm{BD}+23^{\circ} 3130$ & INT & 1991 May & $2 \times 1400$ & 10.540 \\
\hline G166-45 & $\mathrm{BD}+26^{\circ} 2606$ & INT & 1991 Jan. & $1500+640$ & 9.731 \\
\hline G180-58 & & INT & 1991 May & $2 \times 1600$ & 11.318 \\
\hline G182-31 & $\mathrm{BD}+36^{\circ} 2964$ & INT & 1991 May & $2 \times 1400$ & 10.370 \\
\hline G188-30 & & INT & 1990 Oct. & $2 \times 1300+800$ & 11.000 \\
\hline G192-43 & & INT & 1991 Jan & $1500+1260$ & 10.307 \\
\hline G205-42 & $\mathrm{BD}+42^{\circ} 3187$ & INT & 1991 May & $2 \times 1200$ & 9.955 \\
\hline G206-34 & & INT & 1991 May & $3 \times 1500$ & 11.409 \\
\hline G207-5 & $\mathrm{BD}+38^{\circ} 3327$ & NOT & 1991 Aug. & 600 & 7.160 \\
\hline $\mathrm{BD}-13^{\circ} 3442$ & & INT & 1991 May & $3 \times 1500$ & 10.26 \\
\hline $\mathrm{CD}-24^{\circ} 1782$ & & INT & 1991 Jan. & 1800 & 9.93 \\
\hline LP815-43 & & INT & 1991 May & $1100+1500$ & 10.91 \\
\hline W6296 & & INT & 1991 Jan. & $4 \times 1800$ & 10.92 \\
\hline W8296 & & INT & 1991 May & $2 \times 1500$ & 10.68 \\
\hline
\end{tabular}

The table also lists previous measurements by other authors giving $95 \%$ confidence limit error bars ${ }^{2}$.

For about 40 stars of the sample this represents the first spectroscopic study in the region of the Li I feature. The rest have been observed by other authors (mainly by Pilachowski et al. 1993, and Thorburn 1994). The common stars among these works allow a comparison between measurements, constituting a test of consistency and on the validity of the observations. On the measurements presented in Table 2 there are 16, 9 and 3 stars with 2, 3 and 4 independent observations respectively, adding in total 49 pairs of measurements to compare. For a couple of measurements $\left(x_{i} \pm \sigma_{x_{i}}\right)$ and $\left(y_{i} \pm \sigma_{y_{i}}\right)$ of the $i$ star, the difference is $\left(a_{i}, \sigma_{a_{i}}\right)$, being $a_{i}=x_{i}-y_{i}$ and

\footnotetext{
${ }^{2}$ For the measurements by Pilachowski et al. 1993, we compute the errorbars from the SNR values provided by C. Pilachowski (private communication) and using $\sigma=1.6(\mathrm{FWHM} \delta x)^{1 / 2} / \mathrm{SNR}$ (Cayrel 1988), where $\delta x$ is the dispersion per pixel and SNR the signal to noise ratio.
}

$\sigma_{a_{i}}=\sqrt{\sigma_{x_{i}}^{2}+\sigma_{y_{i}}^{2}}$. The quantity $\sum_{i}^{49} a_{i}^{2} / \sigma_{a_{i}}^{2}$ follows approximately a $\chi^{2}$ distribution with 49 degrees of freedom. The variance of the distribution is $\sim 2 \times 49$ which defines the $1 \sigma$ and the $2 \sigma$ level as the ranges $38 \leq \chi^{2} \leq 59$ and $31 \leq \chi^{2} \leq 71$, respectively. In our case $\chi^{2}=116$, which is not in the mentioned ranges and a value extremely unlikely if all measurements were consistent. To test the possible presence of systematic errors in some of the measurements, we have removed from the above comparison the measurements in which $\left|a_{i}\right| / \sigma_{a_{i}} \geq 2.5$. Doing the same analysis than above, the value obtained now is $\chi^{2}=52$ for 41 pairs of measurements, which is in good agreement with the statistical expectations. This seems to indicate that in $\sim 20 \%$ of the common pairs of measurements there are additional sources of error not included in the uncertainty provided by the quoted errorbars. Other statistical tests give the same conclusion. Explicitly we suspect of the following stars: 
Table 2. Photometry and Li I equivalent widths of the programme stars

\begin{tabular}{|c|c|c|c|c|c|c|c|c|c|c|c|}
\hline Star & $V-K$ & $\operatorname{Ref}_{1}$ & $E(B-V)$ & $c_{1}$ & $b-y$ & $E(b-y)$ & {$[\mathrm{Fe} / \mathrm{H}]$} & $\mathrm{Ref}_{2}$ & $\begin{array}{c}\text { EW this work } \\
(\mathrm{m} \AA)\end{array}$ & $\begin{array}{c}\text { EW others } \\
(\mathrm{m} \AA)\end{array}$ & $\operatorname{Ref}_{3}$ \\
\hline HD 4906 & & & & 0.297 & 0.483 & 0.041 & -0.84 & 4 & $\leq 13$ & & \\
\hline HD 24289 & 1.63 & 1 & 0.02 & 0.288 & 0.388 & 0.045 & -1.64 & 5 & $50 \pm 3$ & $49 \pm 6.8,45 \pm 5.2$ & 8,4 \\
\hline HD 45282 & 1.91 & 2 & & 0.277 & 0.451 & 0.029 & -1.51 & 5 & $\leq 16$ & $9.9 \pm 5.2$ & 4 \\
\hline HD 88725 & 1.57 & 3 & 0.00 & 0.325 & 0.409 & & -0.65 & 6 & $\leq 14$ & & \\
\hline HD 101063 & & & & 0.272 & 0.499 & 0.091 & -1.35 & 5 & $11 \pm 3$ & $12.3 \pm 3.9$ & 4 \\
\hline HD 103912 & 2.17 & 1 & 0.00 & 0.306 & 0.527 & & -1.71 & 6 & $\leq 4$ & $\leq 4, \leq 3$ & 8,4 \\
\hline HD 106038 & & & 0.00 & 0.264 & 0.342 & & -1.45 & 6 & $68 \pm 3$ & & \\
\hline HD 108976 & & & & 0.382 & 0.304 & & & & $33 \pm 7$ & & \\
\hline HD 111721 & 2.16 & 1 & & 0.296 & 0.511 & 0.069 & -1.11 & 5 & $23 \pm 2$ & $17.2 \pm 3.5$ & 4 \\
\hline HD 112573 & 1.69 & 3 & 0.00 & 0.250 & 0.420 & & -0.53 & 6 & $\leq 12$ & & \\
\hline HD 126681 & 1.66 & 1 & & 0.191 & 0.400 & 0.008 & -1.16 & 5 & $16 \pm 4$ & & \\
\hline HD 134439 & 2.04 & 2 & 0.00 & 0.165 & 0.484 & & -1.92 & 7 & $\leq 10$ & & \\
\hline HD 134440 & 2.28 & 3 & 0.00 & 0.173 & 0.524 & & -1.52 & 6 & $\leq 6$ & & \\
\hline HD 149414 & & & 0.00 & 0.162 & 0.476 & & -1.54 & 6 & $10 \pm 3$ & & \\
\hline HD 157948 & 2.00 & 3 & 0.00 & & & & -0.71 & 6 & $17 \pm 3$ & & \\
\hline HD 158226 & 1.52 & 3 & 0.00 & 0.312 & 0.389 & & -0.61 & 6 & $\leq 4$ & & \\
\hline HD 161770 & 2.03 & 2 & & 0.301 & 0.489 & & -1.38 & & $42 \pm 3$ & $44.9 \pm 3$ & 4 \\
\hline HD 163810 & 1.91 & 2 & 0.03 & 0.199 & 0.423 & & -1.55 & 6 & $28 \pm 4$ & $22.1 \pm 2.1$ & 4 \\
\hline HD 179626 & 1.58 & 1 & 0.05 & 0.293 & 0.373 & 0.019 & -0.94 & 6 & $27 \pm 2$ & & \\
\hline HD 184448 & & & 0.00 & 0.327 & 0.409 & & -0.61 & 6 & $\leq 9$ & & \\
\hline HD 199476 & & & 0.00 & & & & -0.49 & 6 & $\leq 6$ & & \\
\hline HD 200580 & 1.55 & 3 & & 0.262 & 0.368 & & -1.00 & & $29 \pm 3$ & $32 \pm 4$ & 15 \\
\hline HD 204155 & 1.51 & 3 & 0.00 & & & & -0.80 & 6 & $\leq 13$ & & \\
\hline
\end{tabular}

- G205-42: There are two additional measurements by Pilachowski et al. (1993) and Thorburn (1994). For this star our measurement is in disagreement with the other two.

- HD 111721 and HD 163810: For these stars there is only one additional measurement by Pilachowski et al. (1993), clearly in disagreement with the values reported in this work.

- G88-32: Our measurement $(19 \pm 5 \mathrm{~mA})$ is discrepant with two previous measurements by Hobbs \& Thorburn (1991; $26 \pm 4.4 \mathrm{m \AA})$ and Thorburn (1994; $28 \pm 5.8 \mathrm{m \AA})$.

- G166-45: This is a very intriguing double lined spectroscopic binary. While Hobbs \& Thorburn (1991) and Thorburn (1994) measure $29 \pm 3.6 \mathrm{m \AA}$, our determination gives $36 \pm 3 \mathrm{m \AA}$, in agreement with the measurement by Rebolo et al. (1988; $35 \pm 3 \mathrm{m \AA})$. In the spectrum obtained by Rebolo et al. (their Fig. 1b) it is possible to appreciate a small asymmetry in the red wing of the Li I line. The new spectrum recorded in 1991 January also shows this asymmetry, possibly indicating that the secondary star also has a Li I line.

- BD -13.3442: Here we report $22 \pm 2 \mathrm{m \AA}$, which presents a serious discrepancy of $8 \mathrm{~mA}$ with the measurement of Thorburn (1994; $30 \pm 6.8 \mathrm{m \AA})$. However, our measurement is in better agreement with that obtained by Ryan et al. (1996; $19 \pm 2 \mathrm{m \AA})$.

- LP815-43: We report here $25 \pm 6 \mathrm{~mA}$. The situation here is confused with several measurements giving around $25 \mathrm{m \AA}$ and others close to $15 \mathrm{m \AA}$.

Removing these stars from the statistical analysis, we found $\chi^{2}=26$ for the remaining 31 pair of measurements indicating that the inconsistency of the sample is due to the stars removed. Previous work on this have been done by Ryan et al. (1996) and Deliyannis et al. (1993). For instance, applying the above analysis to the sample analyzed by Ryan et al. we obtain $\chi^{2}=248$ for 148 pair of measurements. Removing 5 stars (Ryan et al. recognized problems in 4 of them) involving in total 10 pair of measurements, the value obtained for $\chi^{2}$ is now compatible with the value expected from the quoted noise of each measurement.

The comparison between our measurements and those by Thorburn (1994) and Pilachowski et al. (1993) is summarized in Fig. 2. Defining $\Delta_{\mathrm{P}}$ and $\Delta_{\mathrm{T}}$ as the mean differences between our measurements and the ones by these authors, respectively, we obtain $\Delta_{\mathrm{P}}=+0.7$ and $\Delta_{\mathrm{T}}=$ $-0.9 \mathrm{m \AA}$, values which show the absence of significant 
Table 2. continued

\begin{tabular}{|c|c|c|c|c|c|c|c|c|c|c|c|}
\hline Star & $V-K$ & $\operatorname{Ref}_{1}$. & $E(B-V)$ & $c_{1}$ & $b-y$ & $E(b-y)$ & {$[\mathrm{Fe} / \mathrm{H}]$} & $\operatorname{Ref}_{2}$ & $\begin{array}{c}\text { EW this work } \\
(\mathrm{m} \AA)\end{array}$ & $\begin{array}{c}\text { EW others } \\
(\mathrm{m} \AA)\end{array}$ & $\operatorname{Ref}_{3}$ \\
\hline HD 215065 & & & 0.00 & & & & -0.61 & 6 & $\leq 8$ & & \\
\hline HD 215257 & 1.51 & 1 & 0.00 & 0.273 & 0.358 & & -0.88 & 6 & $31 \pm 4$ & & \\
\hline HD 216588 & & & 0.00 & & & & -0.76 & 6 & $\leq 12$ & & \\
\hline HD 218209 & & & 0.00 & 0.258 & 0.419 & & -0.64 & 6 & $\leq 7$ & & \\
\hline HD 221613 & & & 0.00 & 0.279 & 0.396 & & -0.55 & 6 & $26 \pm 3$ & & \\
\hline G3-16 & & & & 0.319 & 0.317 & 0.012 & -1.92 & 5 & $33 \pm 4$ & & \\
\hline G16-9 & 2.20 & 3 & 0.00 & 0.247 & 0.514 & & -0.88 & 6 & $\leq 10$ & & \\
\hline G20-15 & 1.83 & 3 & 0.10 & 0.247 & 0.452 & & -1.99 & 6 & $33 \pm 4$ & $28.3 \pm 5.0,35.1$ & 4,11 \\
\hline G24-3 & 1.46 & 2 & 0.02 & 0.271 & 0.363 & 0.022 & -1.81 & 6 & $32 \pm 3$ & $28.5 \pm 4.0$ & 4 \\
\hline G26-9 & 2.83 & 1 & & 0.188 & 0.588 & & -1.19 & 5 & $24 \pm 4$ & & \\
\hline G29-23 & 1.35 & 1 & & 0.332 & 0.339 & 0.012 & -1.75 & 5 & $34 \pm 2$ & 27 & 17 \\
\hline G41-41 & 1.27 & 1 & 0.01 & 0.382 & 0.305 & & -2.80 : & 6 & $22 \pm 6$ & $18 \pm 6.8,20 \pm 3$ & 8,9 \\
\hline G59-27 & 1.35 & 2 & 0.00 & 0.312 & 0.324 & 0.000 & -2.20 & 6 & $28 \pm 5$ & $27 \pm 4.4$ & 8 \\
\hline G60-26 & 1.85 & 1 & & 0.236 & 0.433 & 0.032 & -1.15 & 5 & $17 \pm 4$ & $13.6 \pm 3.5$ & 4 \\
\hline G63-46 & 1.53 & 1 & 0.00 & 0.272 & 0.385 & & -1.03 & 6 & $\leq 15$ & & \\
\hline G64-12 & 1.20 & 3 & 0.00 & 0.336 & 0.312 & 0.034 & -3.38 & 7 & $24 \pm 3$ & $28 \pm 7.2,23 \pm 10,27.6,25,31$ & $8,10,11,12,13$ \\
\hline G64-37 & 1.19 & 1 & 0.01 & 0.329 & 0.299 & 0.013 & -3.38 & 7 & $17 \pm 4$ & $14 \pm 3.2,16 \pm 3.0,18.0,14$ & $8,9,11,14$ \\
\hline G66-22 & 2.02 & 1 & 0.00 & 0.190 & 0.452 & 0.015 & -1.30 & 6 & $20 \pm 4$ & & \\
\hline G69-4A & & & 0.00 & & & & -0.80 & 6 & $\leq 5$ & & \\
\hline G69-4B & & & 0.00 & & & & -0.63 & 6 & $\overline{\leq} 5$ & & \\
\hline G74-5 & 1.55 & 3 & 0.00 & 0.335 & 0.389 & & -1.05 & 6 & $11 \pm 3$ & $14 \pm 4$ & 15 \\
\hline G88-27 & 1.38 & 1 & 0.01 & 0.310 & 0.336 & & -1.65 & 6 & $45 \pm 8$ & & \\
\hline G88-32 & 1.28 & 2 & 0.01 & 0.356 & 0.311 & & -2.71 & 6 & $19 \pm 5$ & $28 \pm 5.8,26 \pm 4.4$ & 8,16 \\
\hline G90-3 & 1.57 & 2 & 0.01 & 0.291 & 0.370 & 0.032 & -2.47 & 6 & $44 \pm 4$ & $43.5 \pm 5.2,44 \pm 5.8$ & 4,8 \\
\hline G101-34 & 2.11 & 1 & 0.01 & 0.284 & 0.506 & & -1.98 & 6 & $16 \pm 3$ & & \\
\hline
\end{tabular}

Table 2. continued

\begin{tabular}{|c|c|c|c|c|c|c|c|c|c|c|c|}
\hline Star & $V-K$ & $\operatorname{Ref}_{1}$ & $E(B-V)$ & $c_{1}$ & $b-y$ & $E(b-y)$ & {$[\mathrm{Fe} / \mathrm{H}]$} & $\operatorname{Ref}_{2}$ & $\begin{array}{c}\text { EW this work } \\
(\mathrm{m \AA})\end{array}$ & $\begin{array}{c}\text { EW others } \\
(\mathrm{mA})\end{array}$ & $\operatorname{Ref}_{3}$ \\
\hline G123-9 & 1.71 & 3 & 0.00 & 0.207 & 0.421 & & -1.32 & 6 & $21 \pm 3$ & & \\
\hline G125-13 & & & & 0.231 & 0.355 & 0.002 & -1.39 & 5 & $40 \pm 4$ & & \\
\hline G125-64 & 1.51 & & 0.04 & 0.185 & 0.377 & & -2.13 & 6 & $43 \pm 6$ & $47 \pm 9$ & 8 \\
\hline G140-46 & 2.08 & 1 & 0.00 & 0.141 & 0.474 & & -1.36 & 6 & $\leq 8$ & & \\
\hline G141-19 & 2.05 & 1 & 0.06 & 0.259 & 0.505 & 0.140 & -2.42 & 7 & $34 \pm 3$ & $34 \pm 4,32.5 \pm 5.0$ & 8,4 \\
\hline G141-47 & 1.57 & 3 & 0.1 & 0.288 & 0.390 & 0.1 & -1.34 & 6 & $32 \pm 4$ & $35 \pm 3.9$ & 4 \\
\hline G166-45 & 1.37 & 2 & 0.00 & 0.280 & 0.336 & & -2.58 & 6 & $36 \pm 3$ & $29 \pm 3.6,35 \pm 3,29 \pm 4.4$ & $8,15,16$ \\
\hline G180-58 & 1.90 & 2 & 0.00 & 0.104 & 0.488 & & -2.36 & 6 & $\leq 10$ & & \\
\hline G182-31 & & & 0.01 & & & & -2.53 & 6 & $2 \overline{5} \pm 3$ & $26 \pm 3.8$ & 8 \\
\hline G188-30 & 1.87 & 1 & 0.01 & 0.130 & 0.464 & & -1.86 & 6 & $11 \pm 3$ & & \\
\hline G192-43 & 1.32 & 1 & 0.01 & 0.304 & 0.322 & & -1.49 & 6 & $73 \pm 8$ & & \\
\hline G205-42 & 1.80 & & 0.00 & 0.231 & 0.430 & 0 & -2.18 & & $15 \pm 4$ & $23 \pm 4.8,24.0 \pm 4.2$ & 8,4 \\
\hline G206-34 & 1.36 & 3 & 0.04 & 0.263 & 0.342 & 0.035 & $-2.88:$ & 6 & $28 \pm 4$ & $27 \pm 5.0$ & 8 \\
\hline G207-5 & & & 0.00 & & & & -0.43 & 6 & $33 \pm 3$ & & \\
\hline $\mathrm{BD}-13^{\circ} 3442$ & & & 0.01 & 0.413 & 0.311 & & -3.14 & 7 & $22 \pm 2$ & $30 \mathrm{~s} \pm 6.8,19 \pm 2$ & 8,9 \\
\hline LP815-43 & & & 0.04 & & & & -3.20 & 7 & $25 \pm 6$ & $22 \pm 4.2,27.0,15$ & $8,13,14$ \\
\hline $\mathrm{CD}-24^{\circ} 1782$ & & & 0.023 & 0.287 & 0.466 & & -2.56 & 5 & $8 \pm 4$ & $12.6 \pm 3.5$ & 4 \\
\hline W6296 & 1.33 & 1 & & 0.348 & 0.330 & 0.017 & -2.54 & 5 & $25 \pm 3$ & & \\
\hline W8296 & 1.72 & 1 & & 0.183 & 0.415 & 0.019 & -1.53 & 5 & $21 \pm 4$ & & \\
\hline
\end{tabular}

Note: $\operatorname{Ref}_{1}, \operatorname{Ref}_{2}$ and $\operatorname{Ref}_{3}$ give references for the values quoted for $(V-K),[\mathrm{Fe} / \mathrm{H}]$ and EW others, respectively.

References:

(1) Alonso et al. 1994. (2) Carney 1983. (3) Laird et al. 1988. (4) Pilachowski et al. 1993. (5) Schuster \& Nissen 1988, 1989. (6) Carney et al. 1987; Laird et al. 1988. (7) Ryan et al. 1991. (8) Thorburn 1994. (9) Ryan et al. 1996. (10) Rebolo et al. 1987. (11) Spite et al. 1996. (12) Spite et al. 1987. (13) Spite et al. 1993. (14) Norris et al. 1994. (15) Rebolo et al. 1988. (16) Hobbs \& Thorburn 1991. (17) Spite et al. 1984. 
systematic effects between the three sets of measurements. The rms of the differences are 4.4 and $4.6 \mathrm{m \AA}$ for the comparison with the samples of Pilachowski et al. and Thorburn, respectively, values which are slightly larger than the expectations in terms of the error bars $(\sim 2.7$ and $\sim 3.5 \mathrm{m \AA}$, respectively). If we remove in the comparison with Thorburn the stars G88-32, G166-45, G205-42, $\mathrm{BD}-13^{\circ} 3442$, and do the same with HD 111721, HD 163810 and G205-42 in the comparison with Pilachowski et al., we found that the rms of the differences were now in perfect agreement with expectations in terms of noise. These results confirm our claims on the inconsistency in $\sim 20 \%$ of the common measurements.

We conclude that for $\sim 20 \%$ of the stars there are systematic effects not taken into account in the reported uncertainties, and we shall bear in mind that any conclusion inferred from the global sample should be robust against rejection of subsamples comprising this fraction of the stars. Repeated independent observations may guarantee a sufficient number of reliable determinations along the $\mathrm{Li}-T_{\text {eff }}$ and $\mathrm{Li}-[\mathrm{Fe} / \mathrm{H}]$ planes to solve the scatter problem. It would be particularly useful to conduct such programme in photometric and spectroscopic twin halo dwarf stars. One possible case of twin stars is that of G64-37 and G64-12. These are the two stars in our sample with the largest number of determinations, all of them being consistent. Our new measurements are in agreement with the best previously estimated values. When our own values are taken into account the new best estimated $\mathrm{Li}$ I EWs are $25.2 \pm 1.2$ and $15.4 \pm 0.9 \mathrm{m \AA}$ for G64-12 and G64-37, respectively. This Li I EW difference between both stars was noted by Ryan et al. (1996) and is reinforced by our new measurement. Given the strong similarity in their $V-K$ and $c_{1}$ indices (see Table 2) we could expect very similar $T_{\text {eff }}$ and gravity and therefore rather different lithium abundances. However, as we can also see in Table 2, when the reddening is taken into account, the stars do not look as similar as was thought. This question will be addressed in more detail in a forthcoming paper.

Acknowledgements. We thank A. Magazzú, J.E. Beckman and E.W. Barnett for their help with the IACUB observations and their support to the project. We also thank C. Pilachowski for providing the $\mathrm{S} / \mathrm{N}$ of their spectra. Finally we wish to thank F. Spite for his support to this project and his useful comments on the manuscript. This work has used the
SIMBAD database. This work has been partially supported by the Spanish DGES under projects PB92-0434-C02 and PB951132-C02-01. This article has been corrected for English and style by Terry Mahoney (Research Division, IAC).

This paper is dedicated to the memory of C.D. McKeith, who contributed much to this work before his untimely death in September 1996.

\section{References}

Alonso A., Arribas S., Martínez-Roger C., 1994, A\&AS 107, 365

Alonso A., Arribas S., Martínez-Roger C., 1996, A\&AS 117, 227

Bonifacio P., Molaro P., 1997, MNRAS 285, 847

Carney B.W., 1983, AJ 88, 623

Carney B.W., Laird J.B., Latham D.W., Kurucz R.L., 1987, AJ 94, 1066

Carney B.W., Latham D.W., 1987, AJ 92, 116

Carney B.W., Latham D.W., Laird J.B., Aguilar L.A., 1994, AJ 107,2240

Cayrel R., 1988, in IAU Symp., 132, Cayrel de Strobel G. \& Spite M. (ed.). Dordrecht Kluwer, p. 345

Deliyannis C.P., Pinsonneault M.H., Duncan D.K., 1993, ApJ 414,740

Deliyannis C.P., Boesgaard A.M., King J.R., 1995, ApJ 452, L13

Hobbs L.M., Thorburn J.A., 1991, ApJ 375, 116

Laird J.B., Carney B.W., Latham D.W., 1988, AJ 95, 1843

McKeith C.D., García López R.J., Rebolo R., et al., 1993, A\&A 273, 331

Molaro P., Primas F., Bonifacio P., 1995, A\&A 295, L47

Norris J.E., Ryan S.G., Stringfellow G., 1994, ApJ 423, 386

Pilachowski C.A., Sneden C., Booth J., 1993, ApJ 407, 699

Rebolo R., Beckman J.E., Molaro P., 1987, A\&A 172, L17

Rebolo R., Beckman J.E., Molaro P., 1988, A\&A 192, 192

Ryan S.G., Norris J.E., Bessell M.A., 1991, AJ 102, 303

Ryan S.G., Beers T.C., Deliyannis C.P., Thorburn J.A., 1996, ApJ 458, 543

Schuster W.J., Nissen P.E., 1988, A\&AS 73, 225

Schuster W.J., Nissen P.E., 1989, A\&A 222, 69

Spite M., Francois P., Nissen P.E., Spite F., 1996, A\&A 307, 172

Spite M., Maillard J.P., Spite F., 1984, A\&A 141, 56

Spite F., Spite M., 1982, A\&A 115, 357

Spite F., Spite M., 1993, A\&A 279, L9

Spite M., Spite F., Peterson R.C., Chaffee F.H. Jr., 1987, A\&A 172, L9

Thorburn J.A., 1994, ApJ 421, 318 